\title{
PERSPECTIVE
}

\section{Testing the 10 most wanted: a preclinical algorithm to screen candidate opioid use disorder medications}

\author{
Matthew L. Banks ${ }^{1}$, E. Andrew Townsend ${ }^{1}$ and S. Stevens Negus ${ }^{1}$ \\ Neuropsychopharmacology (2019) 44:1011-1012; https://doi.org/10.1038/s41386-019-0336-5
}

\begin{abstract}
The National Institute on Drug Abuse Division of Therapeutics and Medical Consequences (NIDA DTMC) recently identified its "10 Most Wanted" drug mechanisms for development of novel medications to treat opioid use disorder (OUD) [1]. In our view, a key step in efficiently evaluating candidate OUD medications will be identification of a preclinical testing algorithm that is both sensitive to approved medications (methadone, buprenorphine, naltrexone) and selective for those positive controls in comparison to negative controls known to be clinically ineffective (see below). Here we propose such an algorithm specify, effect profiles that could be used to prioritize candidates for clinical testing, and advocate for building a publicly accessible database as research progresses.
\end{abstract}

Figure 1 shows the proposed two-track testing algorithm. This algorithm is founded on four principles of medications development described previously by us [2-4] and others [5]. These principles are based primarily on results from studies in nonhuman primates, but these principles are increasingly being extended to studies in rats as well. (1) Candidate medications should decrease opioid self-administration. Human opioid misuse is a learned operant behavior best modeled preclinically using opioid self-administration procedures. (2) Decreases in opioid selfadministration should be sustained during chronic treatment. Acute dosing studies can be useful to determine potency and time course of candidate-medication effects; however, OUD medications are administered chronically, and preclinical studies should evaluate medication effectiveness to produce sustained decreases in opioid self-administration during chronic medication treatment. (3) Decreases in opioid self-administration should be selective. Candidate medications can decrease opioid self-administration either by decreasing sensitivity to the reinforcing effects of the self-administered opioid (the desired effect) or by producing nonselective decreases in behavior due to sensory, cognitive, or motor impairment (undesired effects). To protect against false-positive outcomes due to undesired effects, it is important to compare candidate-medication effects on opioid self-administration with effects on responding maintained by a non-opioid reinforcer (e.g., food). This can best be accomplished using choice procedures to indicate not only if a candidate medication decreases opioid selfadministration, but also if it promotes a sustained and clinically desirable reallocation of behavior away from opioid use and toward more adaptive responding maintained by alternative nondrug reinforcers. (4) Medications should be targeted for use in either dependent or non-dependent subjects. OUD medications are used clinically in patients with graded degrees of opioid physical dependence, and the degree of dependence is a critical determinant of medication effectiveness. For example, methadone is used clinically only in highly opioid-dependent patients, and preclinically, it produces selective decreases in opioid selfadministration only in dependent subjects undergoing withdrawal. Conversely, naltrexone is used primarily in non-dependent patients, and preclinically, it selectively decreases opioid selfadministration only in non-dependent subjects.

In view of these principles, we propose that candidate OUD medications should be tested for their effectiveness to produce sustained and selective decreases in opioid- vs. food-maintained responding in non-dependent subjects (Track 1) or in dependent subjects undergoing withdrawal (Track 2) (see Fig. 1). Track 1 consists of a sequence of studies to assess medication effects in subjects with restricted opioid access insufficient to produce significant physical dependence. Subjects trained to selfadminister an abused opioid (e.g., heroin, fentanyl) under a fixed-ratio or progressive-ratio schedule would be tested with multiple candidate-medication doses first using acute dosing (Step 1) to establish potency and time course of effects, then using repeated/chronic dosing (e.g., daily or continuous treatment for a week, Step 2). Drug self-administration sessions are often scheduled to occur daily, and treatment regimens can be integrated into this schedule of daily sessions; however, intervals between self-administration sessions can also be extended to assess expression and treatment of relapse after longer periods of abstinence. Selectivity of medication effects would be evaluated on rates of food-maintained responding either concurrently with Steps 1 and 2 in the same subjects (if subjects are trained on a multiple schedule with separate components of opioid and food availability) or in separate subjects after effectiveness to produce sustained decreases in opioid self-administration has been demonstrated (Step 3). Lastly, effects of repeated medication delivery on opioid vs. food choice would be determined to evaluate medication effectiveness to promote a reallocation of behavior from opioid- to food-maintained responding (Step 4). Naltrexone would serve as a positive control for this track, and medication priority for promotion to human-laboratory testing in non-dependent subjects would increase with sequential positive outcomes in Steps 1-4.

Track 2 consists of a sequence of studies to assess medication effects in subjects with more extensive opioid access sufficient to produce physical dependence and enable withdrawal-associated increases in opioid self-administration. Specifically, withdrawal promotes relapse in dependent OUD patients, and preclinically, withdrawal in dependent subjects increases breakpoints for opioids under progressive-ratio schedules and choice of opioid vs. food

${ }^{1}$ Department of Pharmacology and Toxicology, Virginia Commonwealth University, Richmond, VA 23298, USA

Correspondence: Matthew L. Banks (matthew.banks@vcuhealth.org) or S. Stevens Negus (sidney.negus@vcuhealth.org)

Received: 10 January 2019 Revised: 29 January 2019 Accepted: 3 February 2019

Published online: 9 February 2019 


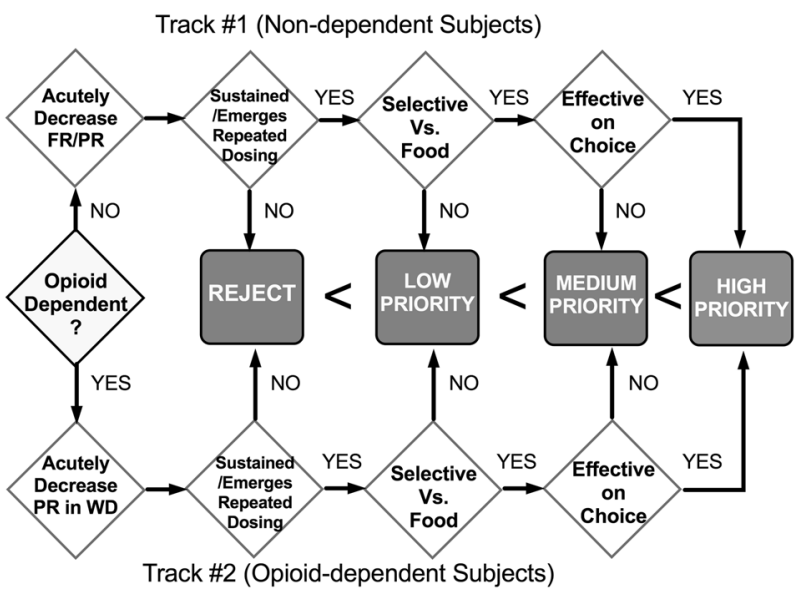

Fig. 1 Proposed preclinical algorithm to evaluate candidate medications for opioid use disorder treatment. The initial decision in the medication development process is to determine whether the candidate medication is directed towards non-dependent individuals (Track\#1) or opioid-dependent individuals (Track\#2). For each Track, compounds would be evaluated through a series of steps that are initially sensitive (e.g., Steps \#1 and 2: are decreases in opioid self-administration observed after acute treatment and sustained or emergent following repeated treatment?) and become progressively more selective (e.g., Step \#4: does repeated treatment decrease opioid choice and produce a reciprocal increase in non-drug alternative choice?). Candidate medications that are effective through all four steps would have the highest priority for promotion to clinical evaluation, whereas candidate medications that fail along the track would have progressively lower priority the earlier in the Track that the failure occurred. Fixed-ratio (FR); Progressive-ratio (PR); Withdrawal (WD)

under choice schedules. Accordingly, candidate-medication effects would be evaluated on withdrawal-associated increases in opioidmaintained breakpoints after acute and repeated administration (Steps 1 and 2, respectively), food-maintained responding (Step 3), and withdrawal-associated increases in opioid vs. food choice (Step 4). Methadone would serve as the positive control for this track, and medication priority for promotion to human-laboratory testing in dependent subjects would again increase with sequential positive outcomes in Steps 1-4.

Buprenorphine could also serve as a positive control in both Track 1 and Track 2. However, repeated buprenorphine treatment sufficient to decrease opioid self-administration may engender physical dependence in non-dependent subjects or produce only partial reversal of withdrawal-associated increases in opioid selfadministration in highly dependent subjects. Negative controls are also essential to validate assay selectivity and provide an empirical framework for interpreting candidate-medication effects, and the field would benefit from selection of a consensus negative control agreed to be ineffective for OUD treatment. We propose clonidine, administered alone, as a negative control in both Track 1 and Track 2. Clinically, clonidine alleviates some sympathomimetic withdrawal signs in dependent OUD patients, and it can reduce relapse risk when used as an adjunct to opioid medications like buprenorphine [6,7]; however, it does not prevent relapse when used as a stand-alone maintenance medication [8]. Preclinically, clonidine produces sedation and motor impairment that can result in non-selective decreases in opioid self-administration typical of many failed candidate medications.

Regardless of the algorithm(s) used to evaluate candidate OUD medications, both the scientific and clinical communities would benefit from a publicly accessible database of results with nonproprietary compounds and with receptor targets of proprietary compounds. This database should include results with methadone, buprenorphine, and naltrexone, and at least one consensus negative control. Such a database, ideally maintained by NIDA DTMC and including data from NIDA DTMC contracts, would facilitate interpretation of results with new candidates, minimize experimental duplication, and guide the field toward promising mechanisms.

\section{FUNDING AND DISCLOSURE}

The authors research was supported by the National Institute on Drug Abuse of the National Institutes of Health under Award Numbers R01DA026946, UH3DA041146, T32DA007027, and F32DA047026. The National Institute on Drug Abuse had no role in the writing or decision to submit the manuscript for publication. The manuscript content is solely the responsibility of the authors and does not necessarily reflect the official views of the National Institutes of Health. All authors declare there are no competing interests or potential conflicts of interest in relation to the research described.

\section{ACKNOWLEDGEMENTS}

We appreciate comments from Dr. Kevin Freeman on an earlier version of the manuscript. We also appreciate the critical comments from the anonymous reviewers.

\section{ADDITIONAL INFORMATION}

Publisher's note: Springer Nature remains neutral with regard to jurisdictional claims in published maps and institutional affiliations.

\section{REFERENCES}

1. Rasmussen K, White DA, Acri JB. NIDA's medication development priorities in response to the Opioid Crisis: ten most wanted. Neuropsychopharmacology, 2018. https://doi.org/10.1038/s41386-018-0292-5.

2. Mello NK, Negus SS. Preclinical evaluation of pharmacotherapies for treatment of cocaine and opioid abuse using drug self-administration procedures. Neuropsychopharmacology. 1996;14:375-24.

3. Negus SS, Banks ML. Medications development for opioid abuse. Cold Spring Harb Perspect Med. 2013;3:a012104.

4. Banks ML, Negus SS. Insights from preclinical choice models on treating drug addiction. Trends Pharmacol Sci. 2017;38:181-94.

5. Czoty PW, Stoops WW, Rush CR. Evaluation of the "pipeline" for development of medications for cocaine use disorder: a review of translational preclinical, human laboratory, and clinical trial research. Pharmacol Rev. 2016;68:533-62.

6. Kowalczyk WJ, et al. Clonidine maintenance prolongs opioid abstinence and decouples stress from craving in daily life: a randomized controlled trial with ecological momentary assessment. Am J Psychiatry. 2015;172:760-7.

7. Mantsch JR, et al. Stress-induced reinstatement of drug seeking: 20 years of progress. Neuropsychopharmacology. 2016;41:335-56.

8. Ling W, et al. A multi-center randomized trial of buprenorphine-naloxone versus clonidine for opioid detoxification: findings from the National Institute on Drug Abuse Clinical Trials Network. Addiction. 2005;100:1090-100. 